\title{
NONPARAMETRIC STATISTICAL MODELS OF GLOBULAR
}

\section{CLUSTER DYNAMICS}

\author{
WILLIAM D. HEACOX \\ University of Hawaii at Hilo, Hilo, HI 96720-4091 USA
}

Observations only partially constrain dynamical models of globular clusters and similar systems, in large part because distances and velocities are seen only in projection, and only at a single (unknown) orbital phase. As a result, some dynamical assumptions are necessary if the cluster dynamics and mass distribution are to be inferred from kinematic data - typically that mass follows light, or that the stars observed are the sole source of the gravitational field in which they move, or that orbital energies are completely thermalized, or that orbital angular momenta are exponentially distributed - most often some combination of these is invoked. Such assumptions, reasonable as they may seem, are almost never justified by the data per se for the cluster in question, and thus diminish the credibility of resulting estimates of cluster dynamics and, especially, of the presence and extent of dark matter.

Apparently one can do better, at least in principle. The use of sophisticated statistical models should allow the determination of dynamical properties of clusters as statistical distributions of orbital energies and angular momenta, even where the individual values are not observable; and of the radial distribution of gravitational potential. This is so because the cluster dynamics and potential are mapped onto the observed kinematics in a completely deterministic manner, given only the assumption of spherical symmetry (an apparently reasonable choice in many cases). The requisite statistical models take the generic form

$$
f_{\mathbf{k}}(\mathbf{k} ; \Phi)=\int f_{\mathbf{k} \mid E, L ; \Phi}(\mathbf{k} \mid E, L ; \Phi) f_{E, L}(E, L) d E d L
$$

where $\mathbf{k}$ is a multivariate kinetic variable, $E$ and $L$ are the orbital specific energy and angular momentum, $\Phi(r)$ is the radial run of gravitational potential within the cluster, and $f$ is the probability density function (pdf) of the indicated variables $\left(f_{x \mid y}\right.$ is the pdf of $x$ conditional upon $y$ ). The left-hand side of this equation is the observed joint statistical distribution of selected kinematic variables (projected velocities and distances) among cluster members; the function $f_{E, L}$ is the underlying true distribution of orbital energies and angular momenta. The integral kernels $f_{\mathbf{k} \mid E, L ; \Phi}$ may be a priori computed from principles of mathematical statistics and the assumption of spherical symmetry. As an example: for the bivariate combination $\mathbf{k}=\left(r_{p}, v_{t}\right)$ of projected distance from the center of the cluster $r_{p}$ and the component of proper motion velocity transverse to the projected radial vector, $v_{t}$, the model kernel is 


$$
\begin{aligned}
& f_{\mathrm{k} \mid E, L ; \Phi}\left(r_{p}, v_{t} \mid E, L ; \Phi\right) \propto \\
& r_{p} \int \frac{d r}{r \sqrt{\left[2(E-\Phi(r))^{2}-(L / r)^{2}\right]\left[r^{2}-r_{p}^{2}\right]\left[(L / r)^{2}-v_{t}^{2}\right]}}
\end{aligned}
$$

When substituted as the integral kernel into Equ. 1 the result is the connection between the observed joint distribution of $r_{p}$ and $v_{t}$, and the underlying gravitational potential and statistical mix of orbital energies and angular momenta. Connections of this sort, for any chosen mixture of observed kinematics $\mathbf{k}$ (projected position, radial velocity, plane-of-sky velocities from individual proper motions), must be satisfied by the same gravitational potential $\Phi(r)$ and (if the observed stars are the same in all cases) the same mixture of orbital energies and angular momenta, $f_{E, L}$. The requisite models (of the form of Equ. 2 for all likely combinations of observable variables) may be found in (Heacox, 1998).

In principle such relations may be numerically inverted to determine the potential and dynamics required to produce the observed joint distributions of all projected kinematics. In practice the calculations appear to require a fast parallel processor and sophisticated optimization algorithms, and there is yet no guarantee that the solution is unique. The computational problem is currently being pursued. As for uniqueness, Heacox (1997) demonstrates that proper motion velocities (available for some clusters) are absolutely required for anything like a unique solution; their absence admits of a mass ambiguity in the solution of at least a factor of 2 . It seems likely that obvious physical constraints - such as non-negative mass densities - will effectively limit the possible solutions to permit, e.g., a resolution of the dark matter problem in spherical globular clusters.

\section{References}

Heacox, W. D.: 1997, Statistical Dynamics of Nonrotating Globular Clusters, Astrophys. J., 490, 263-266

Heacox, W. D.: 1998, Nonparametric Statistical Models of Spherically Symmetric Kinematics, Astrophys.J. Supp. Ser., 114, 121-132 